\title{
Evaluación de competencias genéricas en egresados de tecnología médica de la Universidad de Talca, Chile
}

\author{
Mónica Maldonado-Rojas, Sylvia Vidal-Flores, Paulina Royo-Urrizola, Verónica Gómez-Urrutia
}

Introducción. Las competencias genéricas se refieren a componentes del desempeño profesional que se vinculan a relaciones interpersonales y actitudes que pueden ser cultivadas en la formación. La Universidad de Talca ha definido una línea de formación orientada al desarrollo de éstas, divididas en dos competencias instrumentales, dos interpersonales y una ciudadana. El objetivo fue conocer, a través de egresados y empleadores, el nivel de logro de estas competencias.

Sujetos y métodos. Participaron 31 sujetos de un total de 56 egresados de tecnología médica bajo el modelo del rediseño curricular 'educación basada en competencias' y siete empleadores de un total de 11. Se aplicaron encuestas consultando por desempeños de las cinco competencias genéricas declaradas por la universidad para todos sus egresados. Los resultados fueron analizados por categorías y expresados en porcentaje que refieren al logro de las competencias.

Resultados. Se encontró que para cuatro de cinco competencias, cerca del $70 \%$ de los egresados señalan haberlas alcanzado en un grado alto/muy alto, en tanto sobre el $70 \%$ de los empleadores señalan que las cinco competencias son demostradas en esa categoría.

Conclusión. Los resultados demuestran que el currículo de formación estaría desarrollando las competencias declaradas, lo que permite la formación de un profesional integral.

Palabras clave. Competencias genéricas. Egresados. Empleadores. Evaluación de competencias. Formación profesional.

\section{Assessment of generic skills in graduates of Medical Technology, University of Talca, Chile}

Introduction. Generic skills refer to professional performance components linked to interpersonal relationships and attitudes that can be developed through education. The University of Talca defined a fundamental line of training aimed at developing generic skills, dividing them into instrumental, interpersonal and those related to active citizenship. In this paper, we seek to determine the level in which university graduates actually acquired these competences, through a survey applied to graduates themselves and their employers.

Subjects and methods. Thirty-one subjects participated in a total of 56 graduates of Medical Technology under the model curriculum 'competency-based education' and seven employers. This survey was based on performance indicators corresponding to the five generic competences declared in the professional profile. The results were analyzed by categories related to the achievement of competencies, expressed as a percentage.

Results. It was found that in four out of five competences, over $70 \%$ of graduates consider to have attained them in a high/very high degree, while over $70 \%$ of employers pointed out that all five are demonstrated in this category.

Conclusion. The results show that the curriculum has been successful in developing the competencies declared thus offering a comprehensive professional training.

Key words. Assessment of skills. Employers. Generic skills. Graduates. Professional training.

\section{Introducción}

La actualización de currículo y metodologías de enseñanza-aprendizaje es una tarea que las universidades chilenas han debido abordar en el último tiempo, en el contexto de las transformaciones de la educación superior. Éstas han enfatizado, por una parte, la verificación de los aprendizajes del alumnado y, por otra, la capacidad de las instituciones de educación superior (IES) para establecer criterios claros en relación a los aprendizajes clave que se desea lograr en los egresados [1]. La tendencia hacia una 'sociedad del aprendizaje' plantea la demanda de una mayor clarificación de rol social de las IES, incluyendo la capacidad de hacer explícita su promesa formativa y la utilidad del conocimiento que ge-
Departamento de Bioquímica Clínica e Inmunohematología (M. Maldonado-Rojas); Departamento de Microbiología (S. Vidal-Flores); Facultad de Ciencias de la Salud; Universidad de Talca. Instituto Abate Molina (P. Royo-Urrizola); Universidad de Talca. Facultad de Ciencias Jurídicas y Sociales; Universidad Autónoma de Chile (V. Gómez-Urrutia). Talca, Chile.

Correspondencia:

Prof. Mónica Maldonado Rojas. Departamento de Bioquímica Clínica e Inmunohematología. Facultad de Ciencias de la Salud. Universidad de Talca. Camino Lircay, s/n. Talca, Chile.

E-mail:

mamaldon@utalca.cl

Conflicto de intereses: No declarado.

Competing interests: None declared.

(C) 2015 FEM 
neran hacia la sociedad [2]. El abordaje de la enseñanza superior por competencias se entiende como un conjunto complejo e integrado de conocimientos, habilidades, actitudes y destrezas requeridas para resolver situaciones de trabajo [3]. Este enfoque se caracteriza por formular el currículo no sólo en términos de contenidos, sino que hace explícito lo que se espera que los estudiantes sean capaces de hacer con esos contenidos. Las competencias ponen el acento en la capacidad de los individuos de movilizar recursos cognitivos, emocionales y sociales para afrontar situaciones propias de su desempeño en el mundo del trabajo. Por ello, consideran tanto elementos cognitivos ('saber') como procedimentales ('saber hacer') y actitudinales ('saber ser') $[4,5]$.

El foco en la movilización de recursos y la capacidad de integrar conocimientos para lograr determinados resultados ha permitido que este enfoque ponga de relieve la importancia de los componentes procedimentales y actitudinales en la formación superior. Según Bassi et al [6], éstas pueden identificarse como competencias socioemocionales o genéricas, por tratarse de componentes del desempeño profesional que se vinculan a relaciones interpersonales y actitudes que pueden cultivarse en la formación. Esta categoría engloba habilidades como el trabajo en equipo, la comunicación asertiva, la capacidad de organizar el tiempo y jerarquizar tareas para afrontar situaciones de presión/estrés, entre otras. Según Blanco [7], las competencias genéricas 'facilitan el desarrollo de una verdadera educación integral, puesto que engloban todas las dimensiones del ser humano. Como consecuencia, suponen un referente obligado para superar una enseñanza meramente academicista y orientar nuestra tarea hacia la formación de una ciudadanía crítica y unos profesionales competentes'.

La importancia de estas competencias para el mundo del trabajo ha sido documentada por estudios como el realizado para el Proyecto Tuning América Latina y específicamente, en el contexto chileno, la investigación realizada por el centro MIDE-UC sobre la base de consultas sistemáticas a egresados de IES chilenas, tanto de pregrado como de posgrado, empleadores y responsables de recursos humanos, en donde muestran la importancia atribuida por el mundo laboral a las competencias socioemocionales o genéricas $[1,8]$.

La Universidad de Talca, en el contexto de renovación curricular, comenzó la implementación del rediseño curricular como una decisión corporativa a contar desde el año 2006. La institución definió para todos sus egresados una línea de formación fundamental orientada al desarrollo de cinco competencias genéricas, que se dividieron en instrumentales (habilitan al estudiante en herramientas de trabajo intelectual indispensables para su éxito en la universidad y posterior desempeño profesional), interpersonales (dirigidas a desarrollar en el estudiante las habilidades necesarias para establecer relaciones sociales y de trabajo constructivas y efectivas) y ciudadanas (orientadas a fortalecer en el estudiante el comportamiento democrático y socialmente responsable evidenciado en la contribución activa a la convivencia pacífica y ambientalmente responsable, la participación constructiva en los procesos democráticos, y el respeto a la pluralidad y valoración de las diferencias en el entorno). Para el desarrollo de estas cinco competencias, la estructura del plan de formación considera, en todas las carreras que se imparten en la institución, 11 módulos distribuidos en ocho semestres.

La carrera de tecnología médica en Chile se imparte en las universidades y tiene una duración de cinco años. La Universidad de Talca, en su perfil de egreso, definió 13 competencias profesionales, que se suman a las cinco genéricas establecidas por la institución. También se definieron cuatro dominios de desempeño: asistencial, administración, docencia e investigación [9]. Una forma de evaluar si los nuevos profesionales tienen las competencias genéricas declaradas en el nuevo modelo de formación basado en competencias es a través de la autopercepción de los egresados, en un plazo corto desde que han terminado su programa educativo. El objetivo planteado en este estudio fue conocer el grado de manifestación respecto de las competencias genéricas declaradas en el perfil de egreso de los tecnólogos médicos de la Universidad de Talca, a través de la autopercepción de los egresados y la percepción que tienen los empleadores de las mismas competencias en los nuevos profesionales que se integran al mundo laboral.

\section{Sujetos y métodos}

La población estudiada se obtuvo por un censo de los egresados bajo el modelo del rediseño curricular 'educación basada en competencias', cohorte 2006 a 2008 (56 en el periodo). El estudio consideró al 100\% de los titulados de la carrera de tecnología médica de estas cohortes. A los 56 titulados se les envió la encuesta a través de correo electrónico. Respondieron a la solicitud de opinión un total de 31, muestra representativa al $90 \%$ de intervalo de confianza con un margen de error del $10 \%$. 
Tabla I. Valoración de los egresados del logro de las competencias de la formación fundamental, carrera de tecnología médica de la Universidad de Talca $(n=31)$.

\begin{tabular}{|c|c|c|c|c|}
\hline & & \multicolumn{3}{|c|}{ Grado de logro de competencias } \\
\hline & & Deficiente & Regular & Alto/muy alto \\
\hline \multirow{2}{*}{$\begin{array}{l}\text { Competencias } \\
\text { instrumentales }\end{array}$} & $\begin{array}{l}\text { Comunicar de modo pertinente en forma oral y escrita } \\
\text { en situaciones diversas y propias de su formación profesional }\end{array}$ & $1(3,2 \%)$ & $8(25,9 \%)$ & $22(70,9 \%)$ \\
\hline & $\begin{array}{l}\text { Aplicar herramientas de aprendizaje autónomo } \\
\text { como estrategia para continuar aprendiendo }\end{array}$ & $1(3,2 \%)$ & $7(22,6 \%)$ & $23(74,2 \%)$ \\
\hline \multirow{2}{*}{$\begin{array}{l}\text { Competencias } \\
\text { interpersonales }\end{array}$} & $\begin{array}{l}\text { Desempeñarse colaborativamente en equipos de trabajo mostrando } \\
\text { liderazgo y emprendimiento en los ámbitos económico y social }\end{array}$ & $1(3,2 \%)$ & $8(25,9 \%)$ & $22(70,9 \%)$ \\
\hline & $\begin{array}{l}\text { Lograr eficacia en el uso de habilidades sociales } \\
\text { para establecer relaciones interpersonales adecuadas }\end{array}$ & $1(3,2 \%)$ & $11(35,5 \%)$ & $19(61,3 \%)$ \\
\hline $\begin{array}{l}\text { Competencias } \\
\text { ciudadanas }\end{array}$ & $\begin{array}{l}\text { Discernir en los ámbitos ético profesional, } \\
\text { social, cultural, ambiental y ciudadano }\end{array}$ & $2(6,4 \%)$ & $5(16,2 \%)$ & $24(77,4 \%)$ \\
\hline
\end{tabular}

Se construyó un instrumento de recogida de la información basándose en las competencias genéricas declaradas en el perfil de egreso de la carrera de tecnología médica de la Universidad de Talca y que corresponden a las competencias de la formación fundamental declaradas por la universidad para todos sus egresados (resolución universitaria n. ${ }^{\circ}$ $082 / 2005)$, que fueron revisadas y reformuladas en 2011 (resoluciones universitarias $n .^{\circ} 180 / 2011$ y n. ${ }^{\circ}$ $237 / 2011$ ). Este instrumento, tipo encuesta, estaba constituido por una parte en que se consultaba sobre el área de desempeño del egresado y la dependencia laboral, y una segunda parte con indicadores de desempeño que correspondían a las competencias genéricas declaradas:

- Competencias instrumentales: a) comunicar de modo pertinente en forma oral y escrita en situaciones diversas y propias de su formación profesional; b) aplicar herramientas de aprendizaje autónomo como estrategia para continuar aprendiendo.

- Competencias interpersonales: a) lograr eficacia en el uso de habilidades sociales para establecer relaciones interpersonales adecuadas; b) desempeñarse colaborativamente en equipos de trabajo mostrando liderazgo y emprendimiento en los ámbitos económico y social.

- Competencias ciudadanas: discernir en los ámbitos ético profesional, social, cultural, ambiental y ciudadano.

Se solicitó a los egresados que evaluaran en qué medida la formación universitaria los había preparado en el conjunto de competencias declaradas en el perfil. Los egresados debían responder de acuerdo a una escala de respuesta de 0 a 7 , donde 0 indicaba que la competencia no se enseñó, 1 indica una valoración muy negativa, y 7 , una valoración muy positiva, para cada una de las competencias.

Un instrumento similar que contenía las competencias de la formación fundamental se envió a 11 empleadores de egresados y se les solicitó que valoraran en qué medida las competencias genéricas eran demostradas por los titulados de tecnología médica de la Universidad de Talca. Respondieron siete empleadores $(63,6 \%)$. No se hace diferencia en los empleadores de egresados que respondieron o no a la encuesta.

\section{Metodología para el análisis de resultados}

Para el análisis de los resultados, la escala de respuestas se codificó según el siguiente esquema:

- 0, 1, 2 y 3: deficiente.

- 4 y 5: regular.

- 6 y 7: alto/muy alto.

El criterio para analizar los resultados obtenidos fueron categorías referidas al logro de las competencias. La interpretación de los resultados de la investigación se realizó a través de porcentaje.

\section{Resultados}

Se procesaron un total de 31 encuestas, lo que corresponde a una tasa de respuesta del $54,4 \%$ de los 
Tabla II. Valoración otorgada por empleadores del logro de las competencias de la formación fundamental, en egresados de la carrera de tecnología médica de la Universidad de Talca $(n=7)$.

\begin{tabular}{|c|c|c|c|c|}
\hline & & \multicolumn{3}{|c|}{ Grado logro competencias } \\
\hline & & Deficiente & Regular & Alto/muy alto \\
\hline \multirow{2}{*}{$\begin{array}{l}\text { Competencias } \\
\text { instrumentales }\end{array}$} & $\begin{array}{l}\text { Comunicar de modo pertinente en forma oral y escrita } \\
\text { en situaciones diversas y propias de su formación profesional }\end{array}$ & 0 & $1(14,3 \%)$ & $6(85,7 \%)$ \\
\hline & $\begin{array}{l}\text { Aplicar herramientas de aprendizaje autónomo } \\
\text { como estrategia para continuar aprendiendo }\end{array}$ & 0 & $2(28,6 \%)$ & $5(71,4 \%)$ \\
\hline \multirow{2}{*}{$\begin{array}{l}\text { Competencias } \\
\text { interpersonales }\end{array}$} & $\begin{array}{l}\text { Desempeñarse colaborativamente en equipos de trabajo mostrando } \\
\text { liderazgo y emprendimiento en los ámbitos económico y social }\end{array}$ & 0 & $2(28,6 \%)$ & $5(71,4 \%)$ \\
\hline & $\begin{array}{l}\text { Lograr eficacia en el uso de habilidades sociales } \\
\text { para establecer relaciones interpersonales adecuadas }\end{array}$ & 0 & $2(28,6 \%)$ & $5(71,4 \%)$ \\
\hline $\begin{array}{l}\text { Competencias } \\
\text { ciudadanas }\end{array}$ & $\begin{array}{l}\text { Discernir en los ámbitos ético profesional, } \\
\text { social, cultural, ambiental y ciudadano }\end{array}$ & 0 & 0 & $7(100 \%)$ \\
\hline
\end{tabular}

titulados bajo el modelo de rediseño curricular. El perfil general de la muestra de egresados puede caracterizarse en los términos siguientes. Según el área de desempeño, el 64,5\% se encuentra trabajando en el área asistencial, el $12,9 \%$ se dedica a la investigación, el 9,7\% trabaja en docencia, el 3,2\% en el área administrativa y el 9,7\% en otras áreas. No estaban empleados el 6,5\%. Según el área de dependencia, un 48,4\% de los egresados están ejerciendo en el sector público y un $42 \%$ en el sector privado. De forma independiente trabajan un 3,2\%.

Con relación a la valoración que entregan los egresados del nivel de logro de las competencias consultadas, la tabla I muestra el porcentaje para cada una, según categorías referidas al logro de las competencias. Los resultados, expresados en porcentaje, muestran que para cuatro de las cinco competencias, sobre el $70 \%$ de los egresados señalan haberlas alcanzado en grado alto/muy alto, y sólo una de las competencias interpersonales se valora en este grado con un porcentaje menor al $70 \%$.

Con relación a los empleadores, responden el $63,6 \%$ y el perfil general de la muestra puede caracterizarse en los términos siguientes. Según el área de desempeño, un 71,4\% son empleadores del área asistencial, un 14,2\% del área de docencia e investigación, y un 14,2\% de otra área (laboratorio ambiental). Según el área de dependencia, un 57,2\% son empleadores del sector público y un $42,8 \%$ del sector privado.

La tabla II muestra la valoración otorgada por los empleadores, expresada en porcentaje según categorías, de cómo son demostradas las competen- cias genéricas por los egresados de tecnología médica de la Universidad de Talca. Los resultados obtenidos señalan que sobre el $70 \%$ de los empleadores consideran que las cinco competencias se demuestran en un grado alto/muy alto.

\section{Discusión}

Los cambios acelerados que ocurren en el conocimiento científico y el acceso a múltiples fuentes de información demanda nuevos conocimientos y destrezas por parte de los profesionales, lo que se logra a través del desarrollo de nuevos procesos educativos centrados en el aprendizaje de los estudiantes que les permitan desarrollar competencias para adquirir este nuevo conocimiento a través de procesos autónomos. Con este propósito, la Escuela de Tecnología Médica de la Universidad de Talca, siguiendo las directrices del rediseño curricular de esta institución, ha integrado explícitamente el desarrollo de las competencias genéricas en su proceso de formación. El logro de estas competencias para la primera cohorte del rediseño curricular se ha evaluado en este estudio.

Existen limitaciones del estudio, como son la construcción del instrumento, que se hizo basándose en la declaración de las competencias establecidas en el perfil de egreso de la carrera en evaluación y en la declaración de la institución formadora, sin que haya existido una validación. Una segunda limitación, la autoaplicación del instrumento, no asegura la total comprensión de los indicadores con- 
sultados, y una última limitación es que se desconoce si los que no responden a la encuesta se abstienen por no estar satisfechos con la formación, o bien tendrían una percepción similar a los que respondieron.

La tasa de respuesta obtenida $(54,4 \%)$ se considera adecuada y podría demostrar que la unidad académica mantiene una importante vinculación con sus egresados, realizando esfuerzos asociados a fortalecer el sentido de pertenencia y a considerar la opinión de sus egresados para los procesos de autoevaluaciones para acreditaciones, procesos de validación de perfil de egreso, entre otros. Los resultados también demuestran que todos los dominios de desempeño declarados en el perfil están representados en la muestra.

Con relación a los resultados obtenidos acerca de la percepción de egresados y empleadores del logro de las competencias genéricas, se discuten los resultados en la categoría referida como alto/muy alto, para cada competencia consultada.

Para el indicador 'comunicar de modo pertinente en forma oral y escrita en situaciones diversas y propias de su formación profesional', el 70,9\% de los egresados y el $85,7 \%$ de los empleadores valoran el logro de esta competencia como alta/muy alta. Esta competencia incluye la capacidad de lectura comprensiva, redacción de textos y comunicación oral. Los estudios sobre dos primeras habilidades en Chile sugieren que, si bien el país está en un buen lugar en América Latina, todavía está en un nivel relativamente bajo. Por ejemplo, y tomando como referente la prueba PISA (Programme for International Student Assessment) que se realiza cada tres años por encargo de la OCDE y que tiene por objeto evaluar hasta qué punto los estudiantes cercanos al final de la educación obligatoria (12 años en Chile) han adquirido algunos de los conocimientos y habilidades necesarios para la participación plena en la sociedad del saber, tanto en el año 2009 como en el 2012 Chile obtuvo una puntuación promedio que ubica el país en el nivel 2 de una escala con siete niveles. En este nivel, los estudiantes son capaces de localizar información que satisfaga varios criterios, contrastar información de acuerdo a una determinada característica, comprender el significado de un fragmento específico de texto, identificar información explícita y relacionar el texto con su experiencia personal [10]. La valoración que hicieron los empleadores de la capacidad comunicativa de los egresados de tecnología médica sugiere que ésta se considerada adecuada para un buen desempeño profesional, pero dado que no se cuenta con un diagnóstico de estas habilidades al ingreso para esta cohorte, no puede descartarse que los estudiantes hayan tenido un buen nivel de habilidades comunicativas al ingresar a la educación superior; no obstante, la heterogeneidad de colegios de origen y nivel socioeconómico de los jóvenes sugiere que este resultado no puede atribuirse sólo a un proceso de selección demandante en este ámbito.

Con respecto a la percepción de 'aprendizaje autónomo', en un trabajo realizado a estudiantes de los últimos años de la carrera de ingeniería industrial de la Universidad Tecnológica Nacional Regional de Buenos Aires, que como requisito tuviesen relación con el mundo laboral a través de pasantías, becas, contratos o puestos efectivos en organizaciones o empresas, un 56\% de los estudiantes manifestaron un grado de satisfacción, y un $44 \%$ de insatisfacción, con el logro de esta competencia [11]. En otro estudio realizado en España [12], los resultados encontrados son los siguientes: un 48,6\% de los estudiantes consideran que han adquirido 'bastante' la competencia de aprender con autonomía, un $40,5 \%$ de los estudiantes considera que la ha aprendido 'mucho' y un 10,8\% enfatiza que la ha aprendido 'muchísimo'. En una encuesta realizada a 2.556 estudiantes de pregrado pertenecientes a diez universidades españolas, los resultados muestran un considerable porcentaje de estudiantes que se autoperciben con un nivel medio-bajo en el grado de desarrollo de su capacidad de aprendizaje autónomo [13]. De acuerdo a los resultados obtenidos en nuestra investigación, manifestamos satisfacción porque un $74,2 \%$ de nuestros egresados ponderaron la habilitación de esta competencia en un grado alto/ muy alto, y un $71,4 \%$ de los empleadores la valoraron en el mismo grado, en un contexto de desempeño laboral.

Para el indicador 'desempeñarse colaborativamente en equipos de trabajo mostrando liderazgo y emprendimiento en los ámbitos económico y social', un $70,9 \%$ de los egresados y un $71,4 \%$ de los empleadores valoran el logro de esta competencia como alta/muy alta. Esta competencia implica integrar equipos de trabajo desarrollando habilidades sociales y de autogestión, demostrar compromiso, responsabilidad, empatía y capacidad de decisión, sobre la base de las dimensiones del liderazgo (iniciativa, posicionamiento, resolución de conflictos...). En la sociedad del conocimiento se presta especial atención a las potencialidades del trabajo en equipo y a la construcción conjunta de conocimiento, por lo que las competencias relacionadas con el trabajo colaborativo son particularmente relevantes [14]. El trabajo en equipo aparece como competencia genérica en el 13,28\% de 187 titulaciones analizadas 
en un estudio realizado en universidades españolas con títulos adaptados al Espacio Europeo de Educación Superior en el curso académico 2009-2010 [15]. Por otra parte, las competencias genéricas más ampliamente tratadas en el conjunto de los grados analizados se corresponden con las del informe de Terrón et al [16], que llevaron a cabo una clasificación jerarquizada de las competencias genéricas más repetidas en las ofertas de empleo con independencia del perfil profesional. En ambos grupos, las competencias de trabajo en equipo figuran en tercer lugar. La alta valoración que hicieron los empleadores y egresados para esta competencia pone de manifiesto que durante la formación se ha privilegiado su desarrollo, lo que está acorde con las nuevas concepciones acerca del rol y sentido de la universidad en interacción con el mundo del trabajo.

Para el indicador 'lograr eficacia en el uso de habilidades sociales para establecer relaciones interpersonales adecuadas', el 61,3\% de los egresados y el $71,4 \%$ de los empleadores valoran el logro de esta competencia como alta/muy alta. Esta competencia de carácter socioemocional comprende habilidades como la asertividad, motivación, autocrítica, autoestima, autocontrol, empatía, capacidad de tomar decisiones, de prevención y solución de conflictos, entre otras [17]. Evaluar cada una de estas habilidades resulta difícil, a no ser que se realice desde una perspectiva disciplinaria específica: la psicología. Un estudio [18] en donde se aplicaron baterías de cuestionarios que en su conjunto cumplen la función de predictor de 'inteligencia emocional' -concepto que integra las dimensiones antes mencionadas- concluye que es una necesidad fomentar la educación de la inteligencia emocional en la educación superior, como un medio para que las personas construyan redes sociales adecuadas que les permitan desarrollar proyectos de vida de forma sana y satisfactoria, creando vínculos significativos que garantizan una adecuada convivencia. Es importante mencionar que está acreditado el entrenamiento de habilidades sociales. En un estudio [19] en donde se estrenó a estudiantes de pregrado del área de la salud en las habilidades sociales de comunicación interpersonal, se obtuvo una diferencia significativa más favorable en los grupos experimentales frente a un grupo control. Nuestra experiencia docente indica -lamentablemente sin contar aún con mediciones- que los estudiantes valoran la experiencia de la habilitación en algunas de las dimensiones que componen las habilidades sociales, experimentando alternativas reales de mejorar las relaciones intersubjetivas, un indicador valorado también positivamente por los empleadores.
La competencia 'discernir en los ámbitos ético profesional, social, cultural, ambiental y ciudadano', fue valorada por un $77,4 \%$ de los egresados y un $100 \%$ de los empleadores como alta/muy alta. Sobre esta competencia genérica o socioemocional existe variada bibliografía respecto a su importancia y a lo que se 'debe hacer' y, a la vez, escaso material empírico en torno al proceso y resultados de aprendizajes. El mercado laboral demanda a las IES competencias que no tienen estándares definidos y que están poco operacionalizados. En este sentido es pertinente señalar que en uno de los módulos (ètica, valoración y sociedad) que contribuyen con el desarrollo de esta competencia, se aplicó una encuesta anónima de percepción a 675 estudiantes respecto a sus aprendizajes en el ámbito del 'saber ser' y del saber convivir. Conforme a lo afirmado por Gómez y Royo [20], cuando se preguntó ‘en qué contexto(s) consideras que podrías aplicar lo aprendido?', un $81 \%$ respondió 'en tus decisiones cotidianas en el ámbito personal (familia, amigos, pareja)'; un $87 \%$, 'en la toma de decisiones en la vida profesional'; un $83 \%$, 'en posibles debates o discusiones con mis compañeros o colegas', y un $72 \%$, 'en posibles debates o discusiones con tus profesores' En ese estudio se concluye que los resultados de este ítem muestran que las actividades formadoras de esta naturaleza - por tratarse de una ética aplicada que demanda la movilización de recursos personales- facilitan la transferencia de sus aprendizajes a otros contextos y se constata que, mediante el análisis ético y la aplicación de estrategias de aprendizaje innovadoras, permitió que los estudiantes tuvieran la posibilidad de reexaminar sus convicciones y valores en un ambiente de aprendizaje formal, potenciando la formación de una ciudadanía activa comprometida con principios éticos universales. La valoración del 100\% que entregan los empleadores para esta competencia proporciona antecedentes que permiten afirmar que, realizando los esfuerzos necesarios, este tipo de capacidades es posible cultivarlas y abrigar esperanzas de que sea posible relevar los principios éticos como orientadores de la acción.

En conclusión, al momento de iniciarse el proceso de rediseño curricular en la Universidad de Talca, en un contexto de incertidumbre, resistencia, sin referentes y en una universidad laica, se decide incorporar, para todas las carreras de pregrado, los módulos de formación fundamental que desarrollan las competencias genéricas, los que tendrían la responsabilidad de ir desarrollando las capacidades y que luego, fortalecidas por la formación disciplinar, finalmente dan cuenta de las competencias de- 
claradas. En el caso específico de la carrera de tecnología médica, este primer estudio de valoración de las competencias genéricas declaradas en el perfil de egreso brinda antecedentes para suponer que éstas se han estado desarrollando en el currículo de formación, dado que tanto los egresados como los empleadores tienen una alta valoración de las mismas. Es necesario seguir evaluando y fortaleciendo la enseñanza, considerando que el desarrollo de este tipo de habilidades permite la formación de un profesional integral que, en su condición de persona, contribuye a una sociedad más justa y más humana, en la cual se imbrican la excelencia profesional y la optimización humana, para responder adecuadamente a las exigencias y requerimientos del mercado laboral.

\section{Bibliografía}

1. Beneitone P, Esquetini C, González J, Marty M, Siufi G, Wagenaar R, eds. Reflexiones y perspectivas de la educación superior en América Latina. Informe final Proyecto Tuning América Latina 2004-2007. Bilbao: Universidad de Deusto/ Gröningen; 2007.

2. Light G, Cox R, Calkins S. Learning and teaching in higher education: the reflective professional. London: Sage; 2009.

3. Sladogna GM. La recentralización del diseño curricular. El perfil profesional y la definición de competencias profesionales. Proyecto INET-GTZ. Cooperación Alemana para el Desarrollo. B. Aires: Instituto Nacional de Educación Tecnológica; 2000.

4. Zabala A, Arnau L. Cómo aprender y enseñar competencias. Barcelona: Graó; 2007.

5. Bunk GP. La transmisión de las competencias en la formación y perfeccionamiento profesionales de la RFA. Revista Europea de Formación Profesional 1994; 1; 8-14.

6. Bassi M, Busso M, Urzúa S, Vargas J. Desconectados: habilidades, educación y empleo en América Latina. New York: Banco Interamericano de Desarrollo; 2012.

7. Blanco A. Desarrollo y evaluación de competencias en educación superior. Madrid: Narcea; 2009.

8. MIDE-UC. Percepción de la calidad actual de los titulados y graduados de la educación superior chilena. Informe final. Santiago: Pontificia Universidad Católica de Chile; 2008. URL:
http://www.mineduc.cl/usuarios/1234/File/Publicaciones/ Informes/3estudio_final_estudio_calidad_de_la_educacin_ superior_mide_uc.pdf.

9. Leiva E, Maldonado M, Vásquez M, Vidal S. Proceso de rediseño curricular basado en competencias de la carrera de tecnología médica. Talca: Universidad de Talca; 2006.

10. OCDE. Programme for International Student Assessment (PISA). Technical reports 2009, 2012. URL: http://www. oecd.org/pisa/publicacionesdepisaenespaol.htm.

11. Rodríguez L, Ibáñez M. Herramienta para medición de las competencias genéricas de los futuros ingenieros respecto de las relaciones interpersonales. Revista de Informática Educativa y Medios Audiovisuales 2005; 2: 7-16.

12. Martínez M. Análisis de las competencias desarrolladas en el aprendizaje autónomo y en el presencial: construyendo la autonomía del alumnado universitario. Universidad de Alicante. Revista de Enseñanza Universitaria 2009; 34: 4-14.

13. Ibarra M, Rodríguez G. Aprendizaje autónomo y trabajo en equipo: reflexiones desde la competencia percibida por los estudiantes universitarios. REIFOP 2011; 14: 4.

14. Sánchez-Elvira A, coord. Criterios para la incorporación de las competencias genéricas en las titulaciones de grado: justificación del mapa de competencias genéricas de la UNED. Primer Seminario de Formación para Asesores de Espacio Europeo. Madrid: UNED; 2008. URL: http://portal.uned.es/pls/ portal/docs/page/uned_main/launiversidad/vicerrectorados/ calidad_e internacionalizacion/innovacion_docente/iued/ documentos/propuesta_mapa_competencias_genericas_ uned.pdf.

15. Sánchez-Elvira A. coord. Del diseño a la evaluación en competencias genéricas: análisis empírico e intervención mediante rúbricas. Programa de estudios y análisis. Proyecto EA2009-0102. Madrid: UNED; 2010. URL: http://e-spacio. uned.es/fez/eserv/bibliuned:500768/Memoria_EA20090102_Sanchez-Elvira_et al_UNED.pdf

16. Terrón MJ, Blanco MY, Learreta B, Sáez B, Fernández A, Blanco A, et al. Integración de las competencias genéricas y su evaluación en los estudiantes en los nuevos títulos de grado. EA-2008-0227. Madrid: MEC; 2009.

17. Bisquerra R, Pérez N. Las competencias emocionales. Educación XXI. Facultad de Educación. UNED 2007; 10; 61-82.

18. Extremera N, Fernández P. Inteligencia emocional, calidad de las relaciones interpersonales y empatía en estudiantes universitarios. Clínica y Salud 2004; 15: 117-37.

19. Marín M, León J. Entrenamiento en habilidades sociales: un método de enseñanza-aprendizaje para desarrollar las habilidades de comunicación interpersonal en el área de enfermería. Psicothema 2001; 13: 247-51.

20. Gómez V, Royo P. Ética cívica: aprender de la experiencia vivida. Revista Calidad de la Educación 2012; 13: 205-21. 